\title{
"Long-term effects of Stevia rebaudiana on glucose and lipid profile, adipocytokines, markers of inflammation and oxidation status in patients with metabolic syndrome "
}

\author{
Eva Kassi ${ }^{1}$, George Landis ${ }^{1}$, Aikaterini Pavlaki ${ }^{1}$, George I. Lambrou ${ }^{2}$, Aimilia Mantzou ${ }^{1}$, Ioannis Androulakis ${ }^{1}$, \\ Andreas Giannakou ${ }^{1}$,Eleni Papanikolaou ${ }^{1}$ and George P. Chrousos ${ }^{1}$
}

${ }^{1}$ Division of Endocrinology, Metabolism and Diabetes, University of Athens Medical School, "Eugenideion" Hospital, Athens, Greece. ${ }^{2}$ First Department of Pediatrics, Choremeio Research Laboratory, University of Athens, Athens-Goudi 11527, Greece

Aim

Metabolic syndrome (MetS) is a cluster of interconnected factors that directly increase the risk of diabetes mellitus type 2 (DMT2) and CVD. Stevia rebaudiana (a low calorie natural sweetener) and its compounds are known for anti-hyperglycemic, anti-inflammatory, anti-oxidant, anti-hypertensive effects. In the present study, we aimed to investigate the effect of Stevia rebaduiana on glucose and lipid profile, adipocytokines, markers of inflammation and oxidation status in patients with metabolic syndrome .

Patients and Methods

38 patients (age $47.3 \pm 10.3$ y) $\left(n_{\text {males }}=14, n_{\text {females }}=24\right)$ with MetS (NCEP/ATPIII criteria) were included. Mets patients were following the same low calorie diet and were randomly assigned to consume either a stevia snack $(n=19)$ four times a week (Stevia group) or a sweet of their choice (n=19) once a week (control group), for four months. BMI, waist/hip ratio (W/H), systolic and diastolic blood pressure (sBP, dBP) were measured before and after intervention. Glucose (Glu), triglycerides, cholesterol (LDL,HDL), uric acid, renal and liver functions were determined in Cobas 8000 (Roche). Insulin ( $\mu \mathrm{IU} / \mathrm{mL})$ was measured with CLIA, and HOMA-IR was calculated. Leptin (ng/dL), Plasminogen Activator Inhibitor 1 (pg/ml) (PAI1), IL-6 (pg/ml),ox-LDL (ng/ml), suPAR (pg/ml) were measured with ELISA, HbA1c in DCA BAYER analyzer and total oxidant status : Perox ( $\mu \mathrm{mol} / \mathrm{lt})$ photometrically. The normal distribution of the continuous variables was assessed by Kolmogorov-Smirnov test. The Chi square test was applied to compare qualitative variables between Stevia and control group. Paired t-test and Wilcoxon was used for comparing the variables with normal and non-normal distribution of patients and controls before and after the intervention. To compare variables between the two groups unpaired t-test and Mann-Whitney for variables with normal distribution and non-normal distribution, respectively, was used.

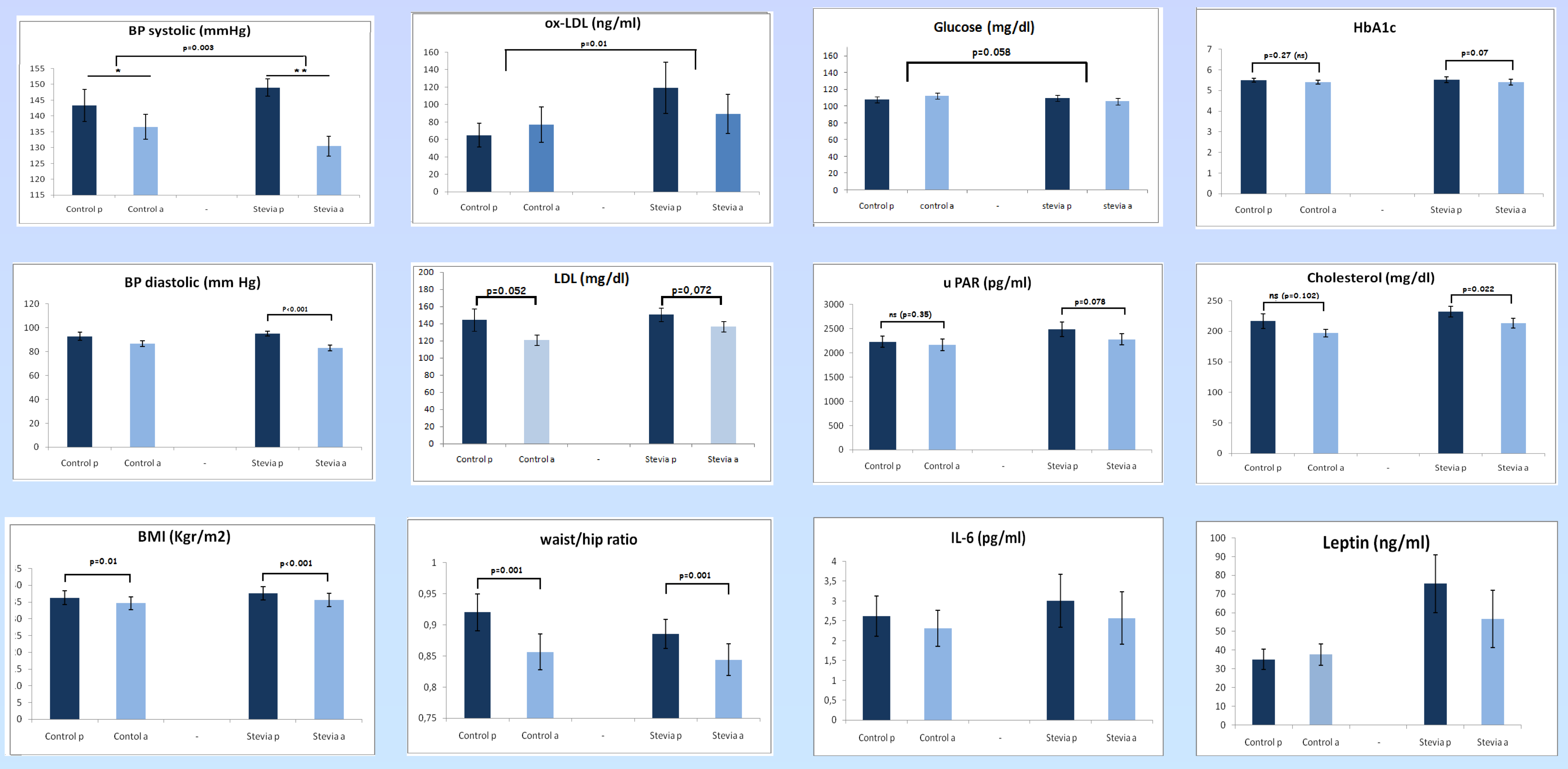

The mean value of the estimated parameters $( \pm$ SEM) before (p) and after (a) the diatery intervention in control group (Control) and in group consumed stevia snack four times per week (Stevia)

Results

After four months dietary intervention, a decrease in BMI $(p=0.01)$ sBP $(p=0.02)$, SGOT $(p=0.004), \gamma G T(p=0.035)$, alk.phospatase $(p=0.008)$ was observed in the control group. Patients in the Stevia group presented significantly lower levels in TCHOL ( $\mathrm{p}=0.022)$, SGOT $(p<0.001), \gamma$-GT $(p<0.001)$, ox-LDL $(p=0.016)$, BMI $(p<0.001)$, sBP $(p<0.001), \mathrm{dBP}(p<0.001)$ and $W / H(p=0.001)$ after four months dietary intervention. A marginally significant decrease in $\operatorname{suPAR}(p=0.078)$, as well as in HbA1c $(p=0.07), L D L(p=0.072)$, and leptin $(p=0.071)$ was also observed. Comparing the changes in serum values, BMI, W/H, BP and HOMA-IR between the two groups over the four months period, patients in Stevia group presented significantly lower levels of ox-LDL $(p=0.01)$ and $s B P(p=0.003)$ and marginally significant decrease in fasting Glu $(p=0.058)$ and leptin $(p=0.054)$ than patients in the control group.

\section{Conclusion}

The introduction of low glycemic load snacks based on Stevia in a low calorie diet in patients with metabolic syndrome proved to be safe and can lead to a further reduction in BP, fasting glucose, ox-LDL and leptin compared to a hypocaloric diet alone, decreasing, thus, further the risk of atherosclerosis and DMT2.

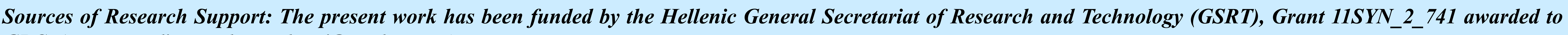
GPC. (corresponding author : ekassi@med.uoa.gr) 